\title{
Évaluation de la teneur en germes gram négatifs des laits et ses conséquences
}

\author{
par \\ Maurice DESFLEURS \\ Ingénieur chimiste I.C.L., docteur $3^{\mathrm{e}}$ cycle \\ (Institut du Lait, des Viandes \\ et de la Nutrition de l'Université de Caen) \\ et Marcel DESFLEURS \\ Pharmacien biologiste
}

La méthode dite " réductase " est utilisée pour le paiement des laits non réfrigérés, suivant leur qualité bactériologique (Arrêté du 16 décembre 1970, J.O. du 6-1-71). Le temps de réduction n'est qu'approximativement fonction inverse du nombre des germes réducteurs, mais ne renseigne pas sur leur nature. Nous avons pensé qu'au moins une indication de groupe serait intéressante. Voici le résumé de nos travaux [3].

\section{I. eSSAIS AVEC LA PENICILLINE}

En ajoutant de la pénicilline au lait, on inhibe les ferments lactiques sans gêner les coliformes, par exemple. On peut espérer obtenir un allongement du temps de réduction pour le lait ayant reçu l'antibiotique.

Nous avons expérimenté sur des laits stérilisés ou tyndallisés ensemencés par les différents ferments lactiques du commerce, par des souches pures, additionnés ou non de doses titrées de 100 en 100 U.I. de pénicilline $\mathrm{G}$ par litre, incubés à $28^{\circ} \mathrm{C}$ puisqu'il s'agit de mésophiles.

De nos nombreux essais, nous concluons qu'il faut généralement 300 U.I. par litre pour empêcher le lait de cailler. Même avec 500 U.I. la réduction du bleu de méthylène n'est pas arrêtée. L'allongement du temps de réduction entre le lait témoin ensemencé et le même ayant reçu la pénicilline, n'est pas significatif. Il est, à l'évidence, 
fonction de l'ensemencement, des espèces utilisées, de leur âge, de leur stade de développement, sans que la reproductibilité soit bonne. On ne peut utiliser la pénicilline pour le but proposé. Elle peut servir dans certains cas [4].

\section{ESSAIS AVEC L'ACIDE NALIDIXIQUE}

L'acide nalidixique ou acide 1-éthyl-1,4 dihydro-7-méthyl-4-oxo-1,8naphtyridine-3-carboxylique est un antibiotique auquel les germes gram positifs ne sont pas sensibles, alors qu'il est actif sur les germes gram négatifs. Il agit in vitro comme in vivo où il est utilisé avec succès pour combattre les infections des voies urinaires. I1 empêcherait la replication de l'A.D.N. bactérien par inhibition de l'A.D.N. polymérase.

Nous relevons dans l'abondante littérature qui lui est consacrée, les concentrations inhibitrices minimales suivantes, en $\mu \mathrm{g}$ par $\mathrm{ml}$ :

E. coli 3 à 7,5 , Proteus $s p$. 2,5 à 20 , Klebsiella $s p$. 2 à 25 , Salmonella $s p .3$ à 50 , Shigella $s p .1$ à 5 , Brucella $s p .7,5$ à 10 , Pseudomonas aeruginosa 4 à 400 , Neisseria gonorrhoeae 0,8 à 3,2, Streptococcus $s p$. 50 à 100.

L'acide nalidixique est également actif sur les Serratia, Moraxella, Enterobacter, Citrobacter, Providencia, Achromobacter, Pseudomonas, etc.

Il y a des différences de sensibilité suivant les germes et, dans chaque espèce, il peut y avoir des souches plus résistantes; leur pourcentage en est faible. Cette résistance n'est pas transférable.

La bactériostase augmente quand le $\mathrm{pH}$ du milieu diminue, ce qui se comprend puisque l'acidité gêne la multiplication des germes gram négatifs.

L'acide nalidixique est soluble dans les bases diluées, mais très malaisement ; très peu dans l'eau pure. Sa solution est stable*. par ml.

Nous avons utilisé l'acide nalidixique à la dose de $0,05 \mathrm{mg}$

Sur chaque lait, on fait une double réduction, dont une de la manière habituelle. Pour l'autre, aux $10 \mathrm{ml}$ de lait, on ajoute également $1 \mathrm{ml}$ de solution de bleu de méthylène de même concentration, soit $5 \mathrm{mg}$, mais contenant en plus $50 \mathrm{mg}$ d'acide nalidixique, le tout pour $100 \mathrm{ml}$ d'eau stérile. On a donc en réalité $0,5 \mathrm{mg}$ de cet acide pour $11 \mathrm{ml}$ de liquide.

\footnotetext{
* Les solutions d'acide nalidixique sont préparées par le Laboratoire Interprofessionnel laitier, rue A.-Fleming - 14200 Hérouville St-Clair, sous la direction de M. Bailleul, que nous tenons à remercier ici bien vivement, ainsi que les Ets Pierre Lanquetot, où bon nombre des expériences citées ont été également faites.
} 


\section{A. Expérimentation avec quelques entérobactéries}

Nous avons d'abord ensemencé du lait écrémé stérilisé avec des cultures purres d'espèces coliformes à la dose d'environ 1 million au $\mathrm{ml}$. Nous avons attribué les résultats peu satisfaisants obtenus à la forte dénaturation du lait provoquée par l'autoclave.

Nous nous sommes alors servi de lait venant d'être trait et tyndallisé par quatre chauffages à $85^{\circ} \mathrm{C}$ pendant $5 \mathrm{mn}$ à $24 \mathrm{~h}$ d'intervalle. Les laits ensemencés sont traités comme ceux prélevés chez les producteurs. Nous avons trouvé les temps de réduction suivants :

$4 \mathrm{~h}$ pour Klebsiella pneumoniae, oxytoca, Enterobacter aerogenes.

4 h 30 pour Escherichia coli, cloacae, Citrobacter.

$7 \mathrm{~h}$ pour Hafniae.

Les tubes qui, parallèlement, avaient reçus en plus l'acide nalidixique étaient toujours bleus après plus de $12 \mathrm{~h}$ à $37^{\circ} \mathrm{C}$.

Nous avons confirmé cette inhibition nette et totale en ensemençant le milieu au désoxycholate pour la recherche des coliformes avec des laits que l'on trouve contenir de 2000 à 30000 de ces germes au $\mathrm{ml}$; si l'on ajoute à ce milieu $50 \mu \mathrm{g}$ d'acide nalidixique au $\mathrm{ml}$, il ne se développe aucune colonie.

\section{B. Expérimentation avec les ferments lactiques}

Nous avons utilisé les ferments lactiques du commerce, vérifiés exempts de coliformes, et également dix souches pures : Streptococcus lactis, cremoris, thermophilus, Lactobacillus lactis, bulgaricus, helveticus, casei, fermenti, plantarum, acidophilus. Nous en avons ensemencé des laits stérilisés à l'autoclave ou tyndallisés comme précédemment. Nous avons procédé à la double réduction sur chacun d'eux.

Les temps de réduction sont souvent égaux, mais généralement on a un léger retard pour les tubes ayant reçu l'acide nalidixique. Il n'est pas constant d'une fois sur l'autre, mais reste dans la limite de $30 \mathrm{mn}$.

I1 n'y a pas inhibition. Nous en avons confirmation en introduisant dans deux boîtes de Pétri la même dilution d'une des cultures pures des souches précitées sur laquelle on coule le même milieu de culture, à la différence que l'un est additionné de $50 \mu \mathrm{g}$ d'acide nalidixique au $\mathrm{ml}$. La numération est la même dans les deux boîtes.

Egalement, sur milieu nutritif ensemencé avec l'une des dix souches pures citées, on pose sur la gélose des disques bio-Mérieux ou Pasteur dosés à $30 \mu \mathrm{g}$ d'acide nalidixique. Quel que soit le ferment lactique, la pousse est uniforme, sans halo d'inhibition autour des disques. 
A la dose employée, l'acide nalidixique n'empêche donc pas la croissance des ferments lactiques. Si l'on a généralement un allongement du temps de réduction, il suffit de ne pas tenir compte d'un retard de $30 \mathrm{mn}$, d'ailleurs peu significatif.

\section{Application pratique}

\section{$1^{\circ}$ Réduction}

On fait une double réduction sur chaque lait, dont une en présence de $50 \mu \mathrm{g}$ d'acide nalidixique par $\mathrm{ml}$, ainsi que nous l'avons indiqué. S'il y a, de ce fait, allongement du temps de décoloration, on l'attribue à l'inhibition des germes gram négatifs, d'où leur évaluation.

Par exemple, sur deux-cent-trente et un laits de producteur, l'augmentation du temps de réduction est :

$\begin{array}{ll}\text { nulle } & \text { pour } 53 \text { soit } 22,94 \mathrm{p} .100 \\ 30 \mathrm{mn} & \text { pour } 27 \text { soit } 11,69 \mathrm{p} .100 \\ 1 \mathrm{~h} & \text { pour } 57 \text { soit } 24,67 \mathrm{p} .100 \\ 1 \mathrm{~h} 30 & \text { pour } 29 \text { soit } 12,55 \mathrm{p} .100 \\ 2 \mathrm{~h} & \text { pour } 21 \text { soit } 9,09 \mathrm{p} .100 \\ \text { de } 2 \text { à } 4 \mathrm{~h} & \text { pour } 36 \text { soit } 15,58 \mathrm{p} .100 \\ 4 \mathrm{~h} \text { et plus } & \text { pour } 8 \text { soit } 3,46 \mathrm{p} .100\end{array}$

Pour quarante-deux autres laits, sans différence entre les deux temps de réduction, 50 p. 100 réduisent le bleu de méthylène en moins de 2 h, 19,04 p. 100 entre 2 et 4 h et 30,95 p. 100 en plus de 4 h. Ces chiffres sont respectivement 35 p. 100,45 p. 100 et 20 p. 100 pour vingt laits présentant un écart de $30 \mathrm{mn}$.

On peut conclure de ces chiffres que dans les laits chargés de germes fortement réducteurs comme les lactiques, l'influence des germes gram négatifs est faible, mais elle se fait sentir dès que leur nombre croît par rapport aux premiers.

Nous avons fait des prélèvements chez soixante-dix producteurs $3 \mathrm{j}$ consécutifs pour trouver, sur ces laits, que le temps de réduction, son allongement, la lacto-fermentation étaient souvent variables. L'augmentation du nombre de germes gram négatifs est liée aux conditions hygiéniques de la traite et de la collecte.

Nos expériences nous ont montré qu'il n'y a pas de corrélation très nette entre le nombre de germes aérobies gram positifs et gram négatifs, déterminées comme nous allons l'indiquer, le nombre de coliformes comptés sur milieu au vert brillant, le temps de réduction, son allongement dû à l'acide nalidixique, la lacto-fermentation, ainsi que l'indique le tableau suivant qui confronte ces différentes analyses faites sur d'autres laits de producteurs (numération au $1 \mathrm{ml}$ ). 


\begin{tabular}{|c|c|c|c|c|c|c|c|}
\hline \multicolumn{3}{|c|}{ Réduction } & \multicolumn{3}{|c|}{ Flore } & \multirow{2}{*}{ Coliformes } & \multirow{2}{*}{$\begin{array}{c}\text { Lacto- } \\
\text { fermentation }\end{array}$} \\
\hline $\begin{array}{c}\text { ordinaire } \\
\mathrm{H}\end{array}$ & $+\underset{H}{\text { Ac. }} \mathrm{N}$ & $\begin{array}{c}\text { différence } \\
\text { H }\end{array}$ & totale & $\begin{array}{c}\text { présumée } \\
\text { G+ }\end{array}$ & $\begin{array}{c}\text { différence } \\
\text { G - }\end{array}$ & & \\
\hline 1.45 & 1.45 & 0 & 1750000 & 1000000 & 750000 & - & Alvéoles \\
\hline 2.30 & 3.30 & 1 & 420000 & 270000 & 150000 & 1250 & Digéré \\
\hline 2.45 & 4. & 1.15 & 620000 & 170000 & 450000 & - & Digéré \\
\hline 3. & 5.30 & 2.30 & 200000 & 120000 & 80000 & 1800 & Alvéoles \\
\hline 4.30 & 7. & 2.30 & 230000 & 140000 & 90000 & 1500 & Gaz \\
\hline 5. & 6. & 1. & 100000 & 50000 & 50000 & 100 & Digéré \\
\hline 5. & 8.30 & 3.30 & 70000 & 20000 & 50000 & 1100 & Gaz \\
\hline 8. & 8.30 & 0.30 & 40000 & 10000 & 30000 & 100 & Gaz \\
\hline
\end{tabular}




\section{Numération}

La qualité bactériologique, principalement pour les laits réfrigérés à la ferme, est basée sur la numération des germes totaux aérobies, suivant l'arrêté précité. On prépare une deuxième boîte de Pétri avec le même milieu additionné de $50 \mu \mathrm{g}$ d'acide nalidixique par $\mathrm{ml}$, sur lequel ne se développent que les colonies de germes réputés gram positifs. La différence entre les deux numérations donne le nombre de germes gram négatifs.

Si pour simplifier on se sert de la seringue munie de l'anse de Burri, on peut en faire le lavage avec la solution titrée d'acide nalidixique pour le comptage des germes gram positifs.

Sur les laits de citernes réfrigérées à la ferme, nous trouvons que le nombre de germes gram négatifs se situe le plus souvent entre 80 et 95 p. 100 des germes totaux aérobies, ce qui confirme ce que l'on savait déjà du développement des psychrotrophes dans ces laits.

\section{CONSEQUENCES ET APPLICATIONS}

Ces méthodes sont utilisées avec satisfaction depuis plus de 1 an par certains laboratoires et fromageries.

Bien que l'on puisse dire que l'acide nalidixique ne sépare pas d'une façon absolue les micro-organismes en deux catégories bien tranchées, il permet facilement une évaluation des germes gram négatifs. Leur présence est toujours l'indice d'une contamination microbienne néfaste.

\section{$1^{\circ}$ Réduction}

La double réduction donne la possibilité d'avertir les producteurs pour qu'ils prennent les précautions d'hygiène adéquates, si le nombre des germes gram négatifs est trop élevé en valeur absolue, mais même pour les laits de première catégorie, s'il est trop grand en valeur relative.

On pourra toujours éliminer les laits qui présentent un écart important et persistant entre les deux temps de réduction.

Dans les laits de fromagerie, il est très important que le rapport germes gram négatifs sur germes totaux soit le plus faible possible. Un chiffre élevé est souvent l'indice de difficultés auxquelles on peut remédier si on l'a déterminé préalablement.

\section{Numération}

Il est bon que les laits réfrigérés, par exemple, n'aient pas plus de 100000 micro-organismes au $\mathrm{ml}$.

Si l'on veut bien, avec nous, demander que la qualité bactériologique des laits réfrigérés soit basée sur le nombre gram négatifs et 
non sur celui des germes totaux comme actuellement - ce que notre méthode rend possible - on pourrait mettre une quantité appropriée de levain dans la citerne réfrigérée à la ferme, avant toute introduction de lait. On peut en espérer une amélioration de la qualité par occupation du milieu.

C'est ce qui se passe si la citerne du camion partant collecter les laits réfrigérés reçoit au départ de l'usine, une dose de levain suffisante pour les ensemencer, comme nous l'avons, nous aussi, préconisé. On a de très bons résultats en fromagerie, même si le lait est pasteurisé dès sa réception.

De même que notre méthode ne se substitue pas à d'autres, telles que numération des psychrotrophes, des coliformes, etc. de même, elle peut être utilisée toutes les fois que le développement de germes gram négatifs est indésirable.

\section{Sélections de certains ferments lactiques}

Autrefois, quand l'on utilisait le lait cru venant d'être réceptionné pour la fabrication de Camembert, on y ajoutait, surtout en hiver, du lait de la veille, conservé en bidons provenant de fermes sélectionnées, pour avoir l'acidité désirée.

Bien que l'élimination des bidons ayant une mauvaise fermentation fut facile, on avait certaines difficultés. Aussi, G. Delsame [1] proposa de faire une lacto-fermentation sur les laits choisis et de se servir de ceux qui présentaient un caillot homogène, même avec quelques bulles de gaz, pour en faire la base d'un levain qui donna de bons résultats.

Il est facile de perfectionner le procédé en incubant vers $28^{\circ} \mathrm{C}$, pour favoriser les lactiques mésophiles, et en faisant une série successive de repiquages dans $10 \mathrm{ml}$ du lait stérile, additionné de $50 \mu \mathrm{g}$ d'acide nalidixique par ml.

On aboutit rapidement à la disparition des micro-organismes nuisibles, par exemple : premier repiquage 166000 coliformes au $1 \mathrm{ml}$; deuxième après 24 h 220 ; troisième néant. La bactériocidie de cet antibiotique est importante quand le milieu est peu éloigné de la neutralité.

La suite des repiquages élimine les ferments lactiques les moins résistants au profit des plus vigoureux, des plus acidifiants. On sait que c'est ce qui se produit avec les ferments du yaourt : domination du Lactobacillus bulgaricus au dépend du Streptococcus thermophilus.

On a fréquemment disparition des lactiques hétéro-fermentaires. Ce n'est pas gênant. Nous pensons, avec G. Delsame, que leur excès est nuisible. Nous venons de voir en fromagerie des gonflements dus à de fortes quantités de leuconostocs et cela même avec du lait 
pasteurisé. Si l'on désire une flore plus complexe, il est facile d'utiliser en complément les levains lactiques du commerce.

On obtient généralement un ferment lactique très acidifiant, par exemple, donnant $70^{\circ} \mathrm{D}$ en $7 \mathrm{~h}$ à $25^{\circ} \mathrm{C}$, alors que dans un lait ensemencé de la même façon, une souche du commerce n'acidifie que vers $30^{\circ} \mathrm{D}$. Ce n'est pas toujours le cas, car un tel lactique peut être absent du lait primitif.

Comme on est parti d'un lait cru contenant fréquemment des bactériophages et que l'on a pratiquement qu'une seule espèce, il n'est pas rare de voir la souche rapidement détruite. Il est donc préférable de recommencer la sélection plutôt que d'entretenir le ferment obtenu.

On peut utiliser les $10 \mathrm{ml}$ du repiquage définitif pour ensemencer $500 \mathrm{ml}$ de levain si l'on adopte le taux maximal de 2 p. 100 , bien que contenant $0,5 \mathrm{mg}$ d'acide nalidixique en tout. Ces $500 \mathrm{ml}$ serviraient à préparer $25 \mathrm{l}$ de levain additionné à $1250 \mathrm{l}$ de lait de fromagerie. La concentration de l'antibiotique s'y retrouverait en quantité parfaitement négligeable. La dose léthale per os en $24 \mathrm{~h}$ est de 4,73 à $3,3 \mathrm{~g} / \mathrm{kg}$ chez la souris. Neuf hommes volontaires ont bien supporté une dose maximale de $8 \mathrm{~g}$ par jour pendant $56 \mathrm{j}$.

On a l'avantage de se servir ainsi de ferments lactiques spécifiques à la région. Ils sont, comme nous l'avons dit [2], un des constituants du cru des produits obtenus.

Par contre, ces ferments lactiques ne sont pas purs. En exemple : un lait à $70^{\circ} \mathrm{D}$ contenant 2 milliards de germes totaux au $\mathrm{ml}$ donne un temps de réduction de $1 \mathrm{mn} 30 \mathrm{~s}$, alors que si l'on avait que des lactiques, il serait inférieur à $30 \mathrm{~s}$. L'acide nalidixique est sans effet sur les germes gram positifs, les levures, les moisissures. Il paraît déconseillé d'utiliser ce levain dans les laits pasteurisés, bien que nous ayons obtenu d'excellents résultats en lait cru.

Certes, on peut employer pour les repiquages des laits spéciaux dans lesquels les bactériophages ne se développent pas, ou encore les additionner d'antibiotiques qui, comme la mycostatine, la pimaricine, sont actifs contre les champignons microscopiques.

Nous constatons que, même en partant du lait d'un même producteur, les résultats ne sont pas constants. Néanmoins, ce procédé peut rendre des services.

\section{Ré s u mé}

Les auteurs montrent que l'addition d'acide nalidixique à la dose de $50 \mu \mathrm{g}$ par $\mathrm{ml}$ de lait inhibe le développement des germes gram négatifs et permet leur évaluation par les méthodes de réduction ou de numération. Il peut être utilisé pour la sélection de levains lactiques. 


\section{S u m m a r y}

The authors point out that the addition of $50 \mu \mathrm{g}$ of nalidixic acid per $1 \mathrm{ml}$ of milk inhibits growth of gram negative species and gives possibility of their valuation by reduction or numeration methods. It may be used for the selection of lactic starters.

Reçu pour publication en décembre 1976.

(Revu et complété en mai 1977).

\section{Bibliographie}

[1] Delsame (G.). - La Tech. Laitière, oct 1968 à mars 1969, nº 606 et suiv.

[2] Desfleurs (M.). - Rev. Laitière Franç., juin 1969, no 266, p. 397-399.

[3] Desfleurs (Maurice), Desfleurs (Marcel). - La Tech. Laitière, 15 janv. 1977, $\mathrm{n}^{\circ}$ 893, p. 11-13.

[4] Simonart (P.), Lambert (R.). - C.R. Congrès Internat. de Laiterie, 1953, T. II, p. 317 . 